Note

\title{
Synthesis, properties and molecular structure of copper(II) and cobalt(II) 1,2-bis(pyrazol-1-ylmethyl)benzene complexes
}

\author{
Wen-Kuen Chang, Gene-Hsiang Lee, Yu Wang, Tong-Ing Ho, Y. Oliver Su*, \\ Yuan-Chuan Lin* \\ Department of Chemistry, National Taiwan University, Taipei, Taiwan, ROC
}

Received by Editor 30 July 1993; received by Publisher 7 March 1994

\begin{abstract}
The synthesis of a new bifunctional ligand, 1,2-bis(pyrazol-1-ylmethyl)benzene (L), and the structures of its complexes with $\mathrm{Cu}(\mathrm{II})$ and $\mathrm{Co}(\mathrm{II})$ ions are described. Ligand $\mathrm{L}$ reacts with copper(II) chloride to form two isomers: the green isomer is $\left[\mathrm{CuLCl}_{2}\right] \cdot 0.5 \mathrm{CH}_{3} \mathrm{OH}(1)$, and the yellowish-green isomer is $\left[\mathrm{Cu}_{2} \mathrm{~L}_{2} \mathrm{Cl}_{4}\right]$ (2). The ligand reacts with cobalt(II) chloride to form a blue complex, $\left[\mathrm{CoLCl}_{2}\right]$ (3). The molecular structures of complexes 1,2 and 3 have been characterized by X-ray diffraction: 1 monoclinic, space group $P 2_{1} / n, Z=8, a=15.465(5), b=13.378(6), c=16.309(6) \AA, \beta=100.98(3)^{\circ} ; 2$ triclinic, space group $P \overline{1}, Z=1, a=8.252(4), b=9.147(8), c=10.414(6) \AA, \alpha=107.05(6), \beta=101.37(4), \quad \gamma=89.26(6)^{\circ} ; 3$ monoclinic, space group $P 2{ }_{1} / c, Z=4, a=10.364(1), b=11.617(3), c=13.720(2) \AA, \beta=109.76(1)^{\circ}$. The coordination sphere of the metal(II) ion in complexes 1 and 3 has a distorted tetrahedral geometry, while that in complex 2 has a distorted square-pyramidal geometry.
\end{abstract}

Keywords: Crystal structures; Copper complexes; Cobalt complexes; Polyfunctional ligand complexes

\section{Introduction}

Polyfunctional ligands derived from pyrazole, imidazole, triazole and pyridazine [1-4] can form transitionmetal complexes in which the metals are brought into close proximity. Molecular structure characterizations of these complexes have been pursued vigorously in many laboratories, because these complexes are used as potential models for biological dimetallic sites [5]. Some dicopper(II) complexes with pyrazolyl ligands have been studied as potential models for hemocyanin by Sorrel and co-workers [6]. Reedijk and co-workers reported that pyrazolyl ligands offered $\mathrm{N}$-donor sites to form halido-bridged copper(II) complexes [1,7]. In this paper, we report the investigation of the complexing properties of the pyrazolyl ligand 1,2-bis(pyrazol-1ylmethyl)benzene $(L)$, which is a bidentate ligand with two N-donor sites on the pyrazolyl rings. The crystal structures of its copper(II) and cobalt(II) complexes, including the monomeric complexes $\left[\mathrm{CuLCl}_{2}\right]$. $0.5 \mathrm{CH}_{3} \mathrm{OH}$ and $\left[\mathrm{CoLCl}_{2}\right]$ and the dimeric complex

\footnotetext{
${ }^{*}$ Corresponding authors.
}

$\left[\mathrm{Cu}_{2} \mathrm{~L}_{2} \mathrm{Cl}_{4}\right]$, have been determined. The spectroscopic and electrochemical properties of these complexes are also reported.

\section{Experimental}

\subsection{Syntheses}

All reagents and solvents were purchased from commercial sources and used as received unless noted otherwise. 1,2-Bis(bromomethyl)benzene was prepared according to the literature method [8].

\subsubsection{1,2-Bis(pyrazol-1-ylmethyl)benzene (L)}

Pyrazole (1.36 g, $20 \mathrm{mmol})$ and sodium carbonate $(2.12 \mathrm{~g}, 20 \mathrm{mmol})$ were added to benzene $(20 \mathrm{ml})$, and then 1,2-bis(bromomethyl)benzene $(2.64 \mathrm{~g}, 10 \mathrm{mmol})$ in benzene $(20 \mathrm{ml})$ was added to the solution. After refluxing for $6 \mathrm{~h}$, the solvents were removed under reduced pressure by rotary evaporation. The residue was washed with acetone and filtered. The product was purified by column chromatography (silica gel 60) using 
ethyl acetate-hexane $(1: 4)\left(R_{\mathrm{f}}=0.51\right)$, giving a pale yellow oil. Yield $1.30 \mathrm{~g}(55 \%)$. Anal. Found: C, 70.21; $\mathrm{H}, 5.96 ; \mathrm{N}, 23.32$. Calc. for $\mathrm{C}_{14} \mathrm{H}_{14} \mathrm{~N}_{4}$ : C, 70.57; $\mathrm{H}, 5.92$; $\mathrm{N}, 23.51 \%$. MS: $m / 2238,170 .{ }^{1} \mathrm{H}$ NMR $\left(\mathrm{CDCl}_{3}\right): \delta=5.30$ $\left(4 \mathrm{H}, \mathrm{s}\right.$, aryl $\left.\mathrm{CH}_{2} \mathrm{~N}\right), 6.23\left(2 \mathrm{H}, \mathrm{m}\right.$, pyrazolyl $\left.\mathrm{C}^{4}-\mathrm{H}\right), 7.05$ $\left(2 \mathrm{H}, \mathrm{m}\right.$, pyrazole $\left.\mathrm{C}^{5}-\mathrm{H}\right), 7.26(4 \mathrm{H}, \mathrm{m}$, aryl $\mathrm{H}), 7.52$ $\left(2 \mathrm{H}, \mathrm{m}\right.$, pyrazolyl $\left.\mathrm{C}^{3}-\mathrm{H}\right)$.

\subsection{2. $\left[\mathrm{CuLCl}_{2}\right] \cdot 0.5 \mathrm{CH}_{3} \mathrm{OH}$ (1) and $\left[\mathrm{Cu}_{2} \mathrm{~L}_{2} \mathrm{Cl}_{4}\right]$ (2)}

Copper(II) chloride dihydrate $(170 \mathrm{mg}, 1 \mathrm{mmol})$ and $\mathrm{L}(238 \mathrm{mg}, 1 \mathrm{mmol})$ were dissolved in methanol $(40$ $\mathrm{ml}$ ), and then the mixture was filtered to remove impurities. Green and yellowish-green crystals were obtained by slow diffusion of diethyl ether into the methanol solution. The green crystals are complex 1 . Anal. Found: C, 44.78; H, 3.98; N, 14.20. Calc. for $\mathrm{C}_{14.5} \mathrm{H}_{16} \mathrm{Cl}_{2} \mathrm{CuN}_{4} \mathrm{O}_{0.5}: \mathrm{C}, 44.80 ; \mathrm{H}, 4.14 ; \mathrm{N}, 14.41 \%$. The yellowish-green crystals are complex 2. Anal. Found: C, 45.14; $\mathrm{H}, 3.83 ; \mathrm{N}, 14.77$. Calc. for $\mathrm{C}_{28} \mathrm{H}_{28} \mathrm{Cl}_{4} \mathrm{Cu}_{2} \mathrm{~N}_{8}$ : C, 45.11; H, 3.98; N, $15.03 \%$.

\subsection{3. $\left[\mathrm{CoLCl}_{2}\right](3)$}

Cobalt(II) chloride hexahydrate $(238 \mathrm{mg}, 1 \mathrm{mmol})$ and $\mathrm{L}(238 \mathrm{mg}, 1 \mathrm{mmol})$ were dissolved in methanol $(40 \mathrm{ml})$, and then the mixture was filtered to remove impurities. Blue crystals were obtained by slow diffusion of diethyl ether into the methanol solution. Anal. Found: C, 45.81; H, 3.86; N, 15.03. Calc. for $\mathrm{C}_{14} \mathrm{H}_{14} \mathrm{Cl}_{2} \mathrm{CoN}_{4}$ : C, 45.68; H, 3.83; N, $15.22 \%$.

\subsection{Physical measurements}

${ }^{1} \mathrm{H}$ NMR spectra were recorded on a Bruker AM$200 \mathrm{WB}$ instrument at $200 \mathrm{MHz}$ using $\mathrm{CDCl}_{3}$ as the solvent, and ESR spectra were recorded on a Bruker ESP $300 \mathrm{X}$-band instrument using diphenylpicrylhydrazyl (dpph) as a standard. Magnetic susceptibilities were measured using the Faraday method on a CAHN 2000 instrument with $\mathrm{Hg}\left[\mathrm{Co}(\mathrm{SCN})_{4}\right]$ as a standard, and diamagnetic corrections were made using Pascal's constants. Mass spectra were recorded on a Finnigan MAT TSQ-46C instrument, and IR spectra on a BIO-RAD FTS-40 spectrometer using $\mathrm{KBr}$ as the support. Elemental analyses were obtained using a Perkin-Elmer 2400 analyzer instrument. Electrochemistry was performed with a Bioanalytical System model CV-27 potentiostat and a BAS X-Y recorder. Cyclic voltammetry (CV) was conducted using a three-electrode cell: a BAS glassy carbon working electrode (with an area of $0.07 \mathrm{~cm}^{2}$ ), a platinum-wire auxiliary electrode and an $\mathrm{Ag} / \mathrm{AgCl}$ (saturated $\mathrm{KCl}$ ) reference electrode; TBAP ((tetra-n-butyl)ammonium perchlorate) was the supporting electrolyte and $\mathrm{CH}_{3} \mathrm{CN}$ was the solvent. The absorption spectra were obtained with a Hewlett-Packard $8452 \mathrm{~A}$ diode-array spectrophotometer.

\subsection{Structure determination}

For the structure determination, suitable crystals of the complexes were chosen. Intensity data were collected at $25{ }^{\circ} \mathrm{C}$ on a CAD-4 diffractometer using monochromated Mo $\mathrm{K} \alpha$ radiation $(\lambda=0.71069 \AA)$. The unitcell constants were derived from a least-squares refinement of 25 setting reflections. The $\theta-2 \theta$ scan technique and a variable scan speed were used to obtain the integrated intensities. Three reference reflections were monitored throughout the measurements; the

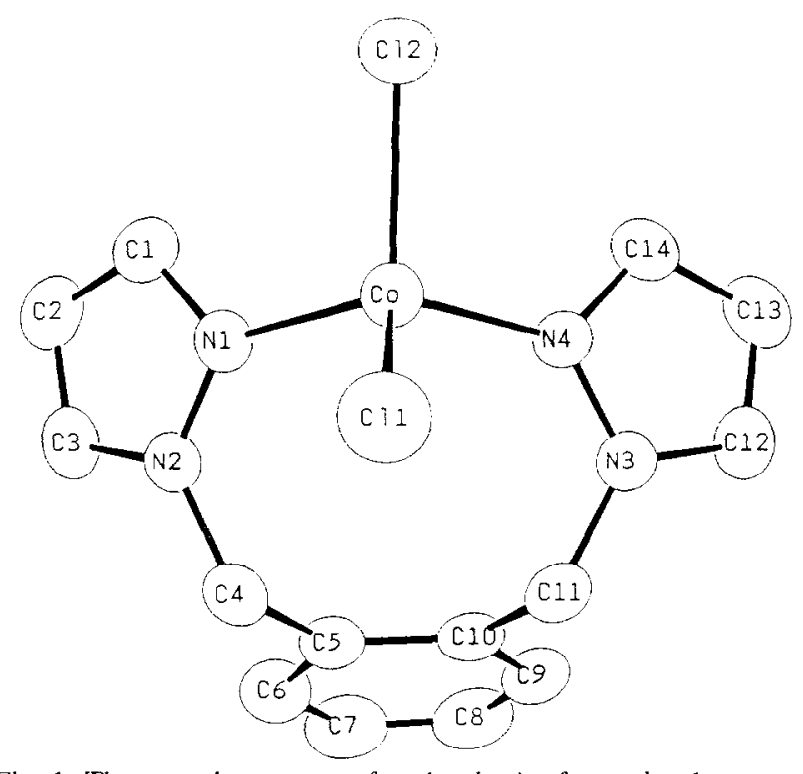

Fig. 1. The crystal structure of molecule A of complex 1.

Table 1

Crystallographic data for complexes 1 and 2

\begin{tabular}{|c|c|c|}
\hline Complex & 1 & 2 \\
\hline Formula & {$\left[\mathrm{CuLCl}_{2}\right] \cdot 0.5 \mathrm{CH}_{3} \mathrm{OH}$} & {$\left[\mathrm{Cu}_{2} \mathrm{~L}_{2} \mathrm{Cl}_{4}\right]$} \\
\hline Molecular weight & 388.76 & 745.49 \\
\hline Crystal size (mm) & $0.40 \times 0.50 \times 0.50$ & $0.13 \times 0.25 \times 0.63$ \\
\hline Crystal & monoclinic & triclinic \\
\hline Space group & $P 2_{1} / n$ & $P \hat{1}$ \\
\hline$a(\AA)$ & $15.465(5)$ & $8.252(4)$ \\
\hline$b(\AA)$ & $13.378(6)$ & $9.147(8)$ \\
\hline$c(\AA)$ & $16.309(6)$ & $10.414(6)$ \\
\hline$\alpha\left(^{\circ}\right)$ & & $107.05(6)$ \\
\hline$\beta\left(0^{\circ}\right)$ & $100.98(3)$ & $101.37(4)$ \\
\hline$\gamma\left({ }^{\circ}\right)$ & & $89.26(6)$ \\
\hline$V\left(A^{3}\right)$ & $3312.4(22)$ & $735.9(8)$ \\
\hline$Z$ & 8 & 1 \\
\hline$F(000)$ & 1584 & 378 \\
\hline$D_{c}\left(\mathrm{Mg} \mathrm{m}^{-3}\right)$ & 1.559 & 1.682 \\
\hline$\mu\left(\mathrm{mm}^{-1}\right)$ & 1.65 & 1.85 \\
\hline $2 \theta$ Range $\left({ }^{\circ}\right)$ & $2-50$ & $2-50$ \\
\hline Ranges of $h, k, l$ & $-16-16,0-14,0-17$ & $-9-9,0-10,-12-11$ \\
\hline Scan parameters & $1.3+0.7 \tan \theta$ & $2.0+0.7 \tan \theta$ \\
\hline Total no. reflections & $4309(3228>2 \sigma)$ & $2.587(2.182>2 \sigma)$ \\
\hline$R, R^{\prime a}$ & $0.038,0.031$ & $0.051,0.068$ \\
\hline$S^{b}$ & 2.80 & 2.24 \\
\hline
\end{tabular}




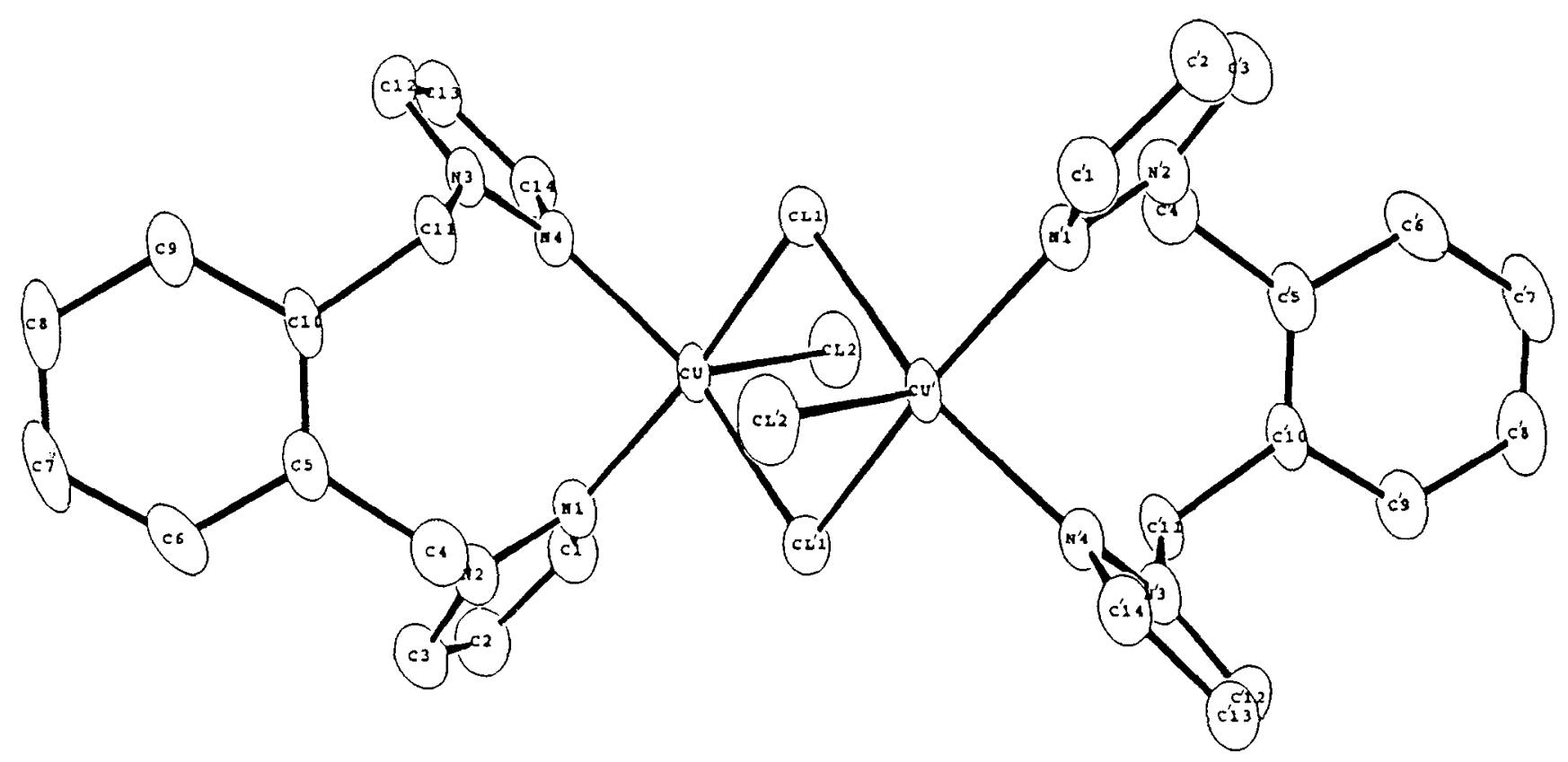

Fig. 2. The molecular structure of complex 2 .

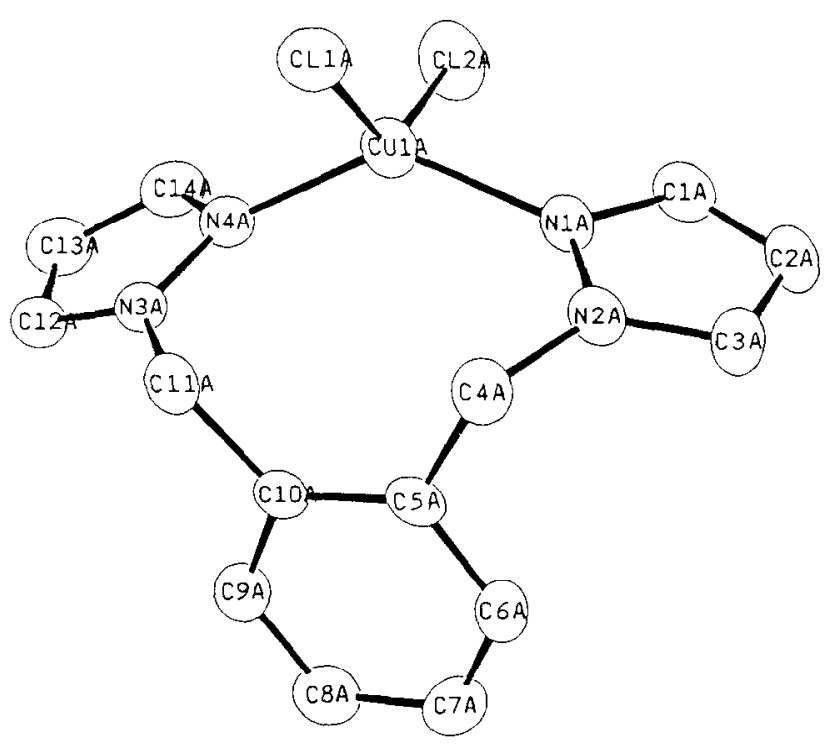

Fig. 3. The molecular structure of complex 3 .

variation of the intensities was less than $2 \%$ for complexes 1 and 3, and less than $6 \%$ for complex 2 . Absorption corrections wcre applied according to the experimental $\Psi$ rotation curve. The molecular structures of complexes 1-3 are shown in Figs. 1-3. Crystal data are given in Tables 1 and 2. Selected bond distances and angles are shown in Tables 3 and 4 .

The structures were solved by the heavy-atom method. The atomic parameters were obtained from the subsequent Fourier syntheses and the least-squares re-
Table 2

Crystallographic data for complex 3

\begin{tabular}{ll}
\hline Formula & {$\left[\mathrm{CoLCl}_{2}\right]$} \\
Molecular weight & 368.13 \\
Crystal size $(\mathrm{mm})$ & $0.40 \times 0.50 \times 0.50$ \\
Crystal & monoclinic \\
Space group & $P 2_{1} / c$ \\
$a(\AA)$ & $10.364(1)$ \\
$b(\AA)$ & $11.617(3)$ \\
$c(\AA)$ & $13.720(2)$ \\
$\beta\left({ }^{\circ}\right)$ & $109.76(1)$ \\
$V\left(\AA^{3}\right)$ & $1554.6(5)$ \\
$Z$ & 4 \\
$F(000)$ & 748 \\
$D_{\mathrm{c}}\left(\mathrm{Mg} \mathrm{m}^{-3}\right)$ & 1.573 \\
$\left.\mu(\mathrm{mm})^{-1}\right)$ & 1.45 \\
$\left.2 \theta \mathrm{Range}^{\circ}\right)$ & $2-50$ \\
Ranges of $h, k, l$ & $-12-11,0-13,0-16$ \\
Scan parameters & $1.3+0.7 \tan \theta$ \\
Total no. reflections & $2740(2208>2 \sigma)$ \\
$R, R^{\prime a}$ & $0.028,0.022$ \\
$S^{\text {a }}$ & 2.26 \\
\hline
\end{tabular}

"Same as in Table 1 .

finements. The final results for the non-hydrogen atoms of complexes 1, 2 and 3 are given in Tables 5, 6 and 7 , respectively. The weighting scheme of the form $1 /\left[\sigma^{2}\left(F_{o}\right)\right]$ was used. All the hydrogen-atom parameters were calculated according to the ideal geometry and were not refined. The structural analyses were carried out on a Microvax III computer using NRCVAX programs [9]. Atomic scattering factors were taken from the literature [10]. 
Table 3

Selected bond distances $(\AA)$ and angles $\left({ }^{\circ}\right)$ of complexes 1 and 2

\section{Complex 1 \\ $\mathrm{Cu}(1 \mathrm{~A})-\mathrm{N}(1 \mathrm{~A})$ \\ $\mathrm{Cu}(1 \mathrm{~A})-\mathrm{N}(4 \mathrm{~A})$ \\ $\mathrm{Cu}(1 \mathrm{~B})-\mathrm{N}(1 \mathrm{~B})$ \\ Cu(1B)-N(4B)}

$\mathrm{Cl}(1 \mathrm{~A})-\mathrm{Cu}(1 \mathrm{~A})-\mathrm{Cl}(2 \mathrm{~A})$

$\mathrm{Cl}(1 \mathrm{~A})-\mathrm{Cu}(1 \mathrm{~A})-\mathrm{N}(4 \mathrm{~A})$

$\mathrm{Cl}(2 \mathrm{~A})-\mathrm{Cu}(1 \mathrm{~A})-\mathrm{N}(4 \mathrm{~A})$

$\mathrm{Cl}(1 \mathrm{~B})-\mathrm{Cu}(1 \mathrm{~B})-\mathrm{Cl}(2 \mathrm{~B})$

$\mathrm{Cl}(1 \mathrm{~B})-\mathrm{Cu}(1 \mathrm{~B})-\mathrm{N}(4 \mathrm{~B})$

$\mathrm{Cl}(2 \mathrm{~B})-\mathrm{Cu}(1 \mathrm{~B})-\mathrm{N}(4 \mathrm{~B})$

\section{Complex 2}

$\mathrm{Cu} \cdot \mathrm{Cu}^{\prime}$

$\mathrm{Cu}-\mathrm{Cl}^{\prime}(1)$

$\mathrm{Cu}-\mathrm{N}(4)$

$\mathrm{Cl}(1)-\mathrm{Cu}-\mathrm{Cl}^{\prime}(1)$

$\mathrm{Cl}(1)-\mathrm{Cu}-\mathrm{N}(1)$

$\mathrm{Cl}(1)-\mathrm{Cu}-\mathrm{Cl}(2)$

$\mathrm{Cl}^{\prime}(1)-\mathrm{Cu}-\mathrm{N}(4)$

$\mathrm{N}(1)-\mathrm{Cu}-\mathrm{N}(4)$

$\mathrm{N}(4)-\mathrm{Cu}-\mathrm{Cl}(2)$

$1.984(4)$
$1.984(4)$
$1.963(4)$
$1.961(4)$
$132.87(7)$
$100.41(13)$
$97.08(13)$
$133.92(8)$
$95.08(13)$
$95.01(13)$

$3.480(3)$

$2.341(3)$

$2.001(6)$

$84.81(9)$

$89.3(2)$

$101.95(9)$

$90.3(2)$

$87.9(2)$

$102.8(2)$
$\begin{array}{lc}\mathrm{Cu}(1 \mathrm{~A})-\mathrm{Cl}(1 \mathrm{~A}) & 2.216(2) \\ \mathrm{Cu}(1 \mathrm{~A})-\mathrm{Cl}(2 \mathrm{~A}) & 2.225(2) \\ \mathrm{Cu}(1 \mathrm{~B})-\mathrm{Cl}(1 \mathrm{~B}) & 2.234(2) \\ \mathrm{Cu}(1 \mathrm{~B})-\mathrm{Cl}(2 \mathrm{~B}) & 2.244(2) \\ \mathrm{Cl}(1 \mathrm{~A})-\mathrm{Cu}(1 \mathrm{~A})-\mathrm{N}(1 \mathrm{~A}) & 100.68(12) \\ \mathrm{Cl}(2 \mathrm{~A})-\mathrm{Cu}(1 \mathrm{~A})-\mathrm{N}(1 \mathrm{~A}) & 97.50(13) \\ \mathrm{N}(1 \mathrm{~A})-\mathrm{Cu}(1 \mathrm{~A})-\mathrm{N}(4 \mathrm{~A}) & 134.24(16) \\ \mathrm{Cl}(1 \mathrm{~B})-\mathrm{Cu}(1 \mathrm{~B})-\mathrm{N}(1 \mathrm{~B}) & 97.25(14) \\ \mathrm{C}(2 \mathrm{~B})-\mathrm{Cu}(1 \mathrm{~B})-\mathrm{N}(1 \mathrm{~B}) & 96.93(13) \\ \mathrm{N}(1 \mathrm{~B})-\mathrm{Cu}(1 \mathrm{~B})-\mathrm{N}(4 \mathrm{~B}) & 148.62(18)\end{array}$

$\mathrm{Cu}-\mathrm{Cl}(1) \quad 2.372(2)$

$\mathrm{Cu}-\mathrm{N}(1) \quad 1.991(6)$

$\mathrm{Cu}-\mathrm{Cl}(2) \quad 2.374(3)$

$\mathrm{Cu}-\mathrm{Cl}(1)-\mathrm{Cu}^{\prime} \quad 95.19(9)$

$\mathrm{Cl}(1)-\mathrm{Cu}-\mathrm{N}(4) \quad 155.2(2)$

$\mathrm{Cl}^{\prime}(1)-\mathrm{Cu}-\mathrm{N}(1)$

$\mathrm{Cl}^{\prime}(1)-\mathrm{Cu}-\mathrm{Cl}(2)$

$\mathrm{N}(1)-\mathrm{Cu}-\mathrm{Cl}(2) \quad 100.2(2)$
Table 4

Selected bond distances $(\AA)$ and angles $\left(^{\circ}\right)$ of complex 3

\begin{tabular}{llll}
\hline $\mathrm{Co}-\mathrm{Cl}(1)$ & $2.2399(9)$ & $\mathrm{Co}-\mathrm{Cl}(2)$ & $2.2395(9)$ \\
$\mathrm{Co}-\mathrm{N}(1)$ & $1.997(2)$ & $\mathrm{Co}-\mathrm{N}(4)$ & $2.011(2)$ \\
$\mathrm{Cl}(1)-\mathrm{Co}-\mathrm{Cl}(2)$ & $115.50(3)$ & $\mathrm{Cl}(1)-\mathrm{Co}-\mathrm{N}(1)$ & $109.60(7)$ \\
$\mathrm{Cl}(1)-\mathrm{Co}-\mathrm{N}(4)$ & $112.71(6)$ & $\mathrm{Cl}(2)-\mathrm{Co}-\mathrm{N}(1)$ & $105.46(7)$ \\
$\mathrm{Cl}(2)-\mathrm{Co}-\mathrm{N}(4)$ & $102.56(6)$ & $\mathrm{N}(1)-\mathrm{Co}-\mathrm{N}(4)$ & $110.62(8)$ \\
\hline
\end{tabular}

\section{Results and discussion}

\subsection{Molecular structures of complexes 1-3}

Complex 1 is a monocopper(II) complex. There is 0.5 methanol molecule as solvent and it is disordered. Each copper(II) ion is coordinated by two nitrogen atoms (N(1), N(4)) from pyrazolyl rings of the ligand $\mathrm{L}$ and two chloride ions $(\mathrm{Cl}(1), \mathrm{Cl}(2))$. The two $\mathrm{Cu}(1 \mathrm{~A})-\mathrm{N}$ distances are both $1.984(4) \AA$, and the two $\mathrm{Cu}(1 \mathrm{~A})-\mathrm{Cl}$ distances are $2.216(2)$ and $2.225(2) \AA$, respectively. However, the $\mathrm{Cu}(\mathrm{BB})-\mathrm{N}$ distances are $1.963(4)$ and 1.961(4) $\AA$, which are shorter than the $\mathrm{Cu}(1 \mathrm{~A})-\mathrm{N}$ distance. All of the $\mathrm{Cu}-\mathrm{N}$ (pyrazole) distances are within the range of other $\mathrm{Cu}-\mathrm{N}$ (pyrazole) distances of four-coordinated $\mathrm{Cu}$ (II) complexes [11,12]. The $\mathrm{Cu}(1 \mathrm{~B})-\mathrm{Cl}$ distances are 2.234(2) and 2.244(2) $\AA$, which are longer than the $\mathrm{Cu}(1 \mathrm{~A})-\mathrm{Cl}$ distances, and the two $\mathrm{Cl}(1)-\mathrm{Cu}-\mathrm{Cl}(2)$ angles are $132.87(7)^{\circ}$ and $133.92(8)^{\circ}$, indicating that the coordination spheres of both copper(II) ions have strongly distorted tetrahedral geometries.
Each copper(II) ion of complex 2 is coordinated by five donors: two pyrazolyl nitrogen atoms (N(1), N(4)) at 1.991(6) and 2.001(6) $\AA$, two bridging chloride ions $\left(\mathrm{Cl}(1), \mathrm{Cl}^{\prime}(1)\right)$ at $2.372(2)$ and 2.341(3) $\AA$ and a nonbridging chloride ion $(\mathrm{Cl}(2))$ at $2.374(3) \AA$ that occupies the fifth coordination site. Two copper(II) ions and two bridging chloride ions form a centrosymmetric planar four-membered ring. The $\mathrm{Cu}-\mathrm{Cl}-\mathrm{Cu}^{\prime}$ bridging angle is $95.19(9)^{\circ}$, and the $\mathrm{Cl}-\mathrm{Cu}-\mathrm{Cl}^{\prime}$ angle is $84.81(9)^{\circ}$. The $\mathrm{Cu} \cdots \mathrm{Cu}^{\prime}$ distance is $3.480(3) \AA$. The coordination geometry is not perfectly square-pyramidal. Addison et al. [13] defined the $\tau$ parameter $(\tau=0$, square-pyramidal geometry; $\tau=1$, trigonal-bipyramidal geometry) to distinguish between a square-pyramidal geometry and a trigonal-bipyramidal geometry. The $\tau$ value for complex 2 is 0.110 , indicating that the structure can be described as having a distorted square-pyramidal geometry. The basal plane involves $\mathrm{N}(1), \mathrm{N}(4), \mathrm{Cl}(1)$ and $\mathrm{Cl}^{\prime}(1)$, while another $\mathrm{Cl}(2)$ occupies the apical position. The $\mathrm{Cu}(\mathrm{II})$ ion is $0.403 \AA$ above the basal plane. In the literature there are several well-characterized examples of fivecoordinated dicopper(II) compounds. Hodgson and coworkers [14] studied a series of these bis $(\mu$-halido)copper(II) complexes and described the structure of these complexes. The distances and angles in complex 2 are normal, compared with the known compounds.

The structure of complex $\mathbf{3}$ is similar to that of complex 1. The two Co-N distances, 1.997(2) and $2.011(2) \AA$, are similar in value $[15,16]$. The two $\mathrm{Co}-\mathrm{Cl}$ distances are $2.2399(9)$ and $2.2395(9) \AA$, respectively. The N(1)-Co-N(4), Cl(1)-Co-Cl(2) and $\mathrm{Cl}(1)-\mathrm{Co}-\mathrm{N}(1)$ angles are $110.62(8)^{\circ}, 115.50(3)^{\circ}$ and $109.60(7)^{\circ}$, re- 
Table 5

Final atomic positional parameters of non-hydrogen atoms for complex 1

\begin{tabular}{|c|c|c|c|c|}
\hline Atom & $x$ & $y$ & $z$ & $\begin{array}{l}B_{\mathrm{eq}}{ }^{\mathrm{a}} \\
\left(\AA^{2}\right)\end{array}$ \\
\hline $\mathrm{Cu}(1 \mathrm{~A})$ & $0.74167(4)$ & $0.33045(5)$ & $0.28718(4)$ & $2.83(3)$ \\
\hline $\mathrm{Cl}(1 \mathrm{~A})$ & $0.82173(10)$ & $0.38433(11)$ & $0.19698(8)$ & $3.47(7)$ \\
\hline $\operatorname{Cl}(2 \mathrm{~A})$ & $0.61423(10)$ & $0.24885(12)$ & $0.27193(11)$ & $4.76(9)$ \\
\hline $\mathrm{N}(1 \mathrm{~A})$ & $0.8182(3)$ & $0.2220(3)$ & $0.34343(24)$ & $2.51(21)$ \\
\hline $\mathrm{N}(2 \mathrm{~A})$ & $0.9045(3)$ & $0.2307(3)$ & $0.37841(25)$ & $2.71(21)$ \\
\hline$N(3 A)$ & $0.7500(3)$ & $0.5398(3)$ & $0.35349(24)$ & $2.61(21)$ \\
\hline $\mathrm{N}(4 \mathrm{~A})$ & $0.6992(3)$ & $0.4595(3)$ & $0.32460(24)$ & $2.61(21)$ \\
\hline$C(1 \mathrm{~A})$ & $0.7992(3)$ & $0.1259(4)$ & $0.3478(3)$ & $3.0(3)$ \\
\hline$C(2 A)$ & $0.8722(4)$ & $0.0734(4)$ & $0.3854(3)$ & $3.6(3)$ \\
\hline$C(3 A)$ & $0.9383(3)$ & $0.1422(4)$ & $0.4048(3)$ & $3.4(3)$ \\
\hline$C(4 A)$ & $0.9477(3)$ & $0.3284(4)$ & $0.3921(3)$ & $2.8(3)$ \\
\hline$C(5 \mathrm{~A})$ & $0.9220(3)$ & $0.3842(4)$ & $0.4647(3)$ & $2.35(24)$ \\
\hline$C(6 \mathrm{~A})$ & $0.9498(3)$ & $0.3418(4)$ & $0.5435(3)$ & $3.1(3)$ \\
\hline$C(7 \mathrm{~A})$ & $0.9313(4)$ & $0.3857(4)$ & $0.6143(3)$ & $3.6(3)$ \\
\hline$C(8 A)$ & $0.8840(1)$ & $0.4734(1)$ & $0.6075(3)$ & $3.5(3)$ \\
\hline$C(9 A)$ & $0.8562(4)$ & $0.5169(4)$ & $0.5298(3)$ & $3.4(3)$ \\
\hline$C(10 A)$ & $0.8751(3)$ & $0.4738(4)$ & $0.4579(3)$ & $2.35(24)$ \\
\hline$C(11 A)$ & $0.8455(3)$ & $0.5314(4)$ & $0.3769(3)$ & $2.8(3)$ \\
\hline$C(12 A)$ & $0.7004(4)$ & $0.6191(4)$ & $0.3635(3)$ & $3.6(3)$ \\
\hline$C(13 A)$ & $0.6142(4)$ & $0.5913(4)$ & $0.3395(3)$ & $4.2(3)$ \\
\hline$C(14 A)$ & $0.6169(3)$ & $0.4922(4)$ & $0.3161(3)$ & $3.5(3)$ \\
\hline $\mathrm{Cu}(\mathrm{BB})$ & $0.69942(5)$ & $0.32946(5)$ & $0.87452(4)$ & $3.57(4)$ \\
\hline $\mathrm{Cl}(1 \mathrm{~B})$ & $0.63329(15)$ & $0.30195(15)$ & $0.74231(11)$ & $8.54(13)$ \\
\hline $\mathrm{Cl}(2 \mathrm{~B})$ & $0.66348(9)$ & $0.29710(11)$ & $0.99883(9)$ & $3.70(7)$ \\
\hline $\mathrm{N}(1 \mathrm{~B})$ & $0.7911(3)$ & $0.2268(3)$ & $0.8805(3)$ & $3.62(24)$ \\
\hline$N(2 B)$ & $0.8642(3)$ & $0.2245(3)$ & $0.9411(3)$ & $3.39(23)$ \\
\hline$N(3 B)$ & $0.7107(3)$ & $0.5411(3)$ & $0.92785(24)$ & $2.89(21)$ \\
\hline$N(4 B)$ & $0.6682(3)$ & $0.4716(3)$ & $0.8740(3)$ & $3.00(22)$ \\
\hline$C(1 B)$ & $0.7911(4)$ & $0.1423(4)$ & $0.8386(4)$ & $4.7(3)$ \\
\hline$C(2 B)$ & $0.8643(5)$ & $0.0861(5)$ & $0.8726(4)$ & $6.0(4)$ \\
\hline$C(3 B)$ & $0.9096(4)$ & $0.1408(4)$ & $0.9372(4)$ & $4.9(3)$ \\
\hline$C(4 B)$ & $0.8913(3)$ & $0.3114(4)$ & $0.9923(3)$ & $3.2(3)$ \\
\hline$C(5 B)$ & $0.9101(3)$ & $0.4002(4)$ & $0.9398(3)$ & $2.5(3)$ \\
\hline $\mathrm{C}(6 \mathrm{~B})$ & $0.9753(3)$ & $0.3870(4)$ & $0.8935(3)$ & $3.5(3)$ \\
\hline$C(7 B)$ & $0.9975(4)$ & $0.4621(4)$ & $0.8436(3)$ & $3.7(3)$ \\
\hline$C(8 B)$ & $0.9546(4)$ & $0.5528(4)$ & $0.8398(3)$ & $3.7(3)$ \\
\hline $\mathrm{C}(9 \mathrm{~B})$ & $0.8892(4)$ & $0.5662(4)$ & $0.8844(3)$ & $3.3(3)$ \\
\hline$C(10 B)$ & $0.8657(3)$ & $0.4914(4)$ & $0.9357(3)$ & $2.5(3)$ \\
\hline$C(11 B)$ & $0.7934(3)$ & $0.5166(4)$ & $0.9840(3)$ & $2.8(3)$ \\
\hline$C(12 B)$ & $0.6679(4)$ & $0.6288(4)$ & $0.9168(4)$ & $4.2(3)$ \\
\hline$C(13 B)$ & $0.5955(4)$ & $0.6157(4)$ & $0.8556(4)$ & $4: 8(3)$ \\
\hline$C(14 B)$ & $0.5979(4)$ & $0.5178(4)$ & $0.8307(3)$ & $3.8(3)$ \\
\hline C & $0.1677(5)$ & $0.1483(6)$ & $0.0171(5)$ & $8.12(22)$ \\
\hline 0 & $0.0917(6)$ & $0.1735(7)$ & $0.0528(5)$ & $6.56(23)$ \\
\hline $\mathbf{O}^{\prime}$ & $0.1446(8)$ & $0.2245(9)$ & $-0.0135(7)$ & $10.8(4)$ \\
\hline
\end{tabular}

${ }^{a} B_{\mathrm{eq}}$ is the mean of the principal axes of the thermal ellipsoid.

spectively. The angles at the cobalt(II) ion clearly indicate a slight distortion away from the ideal tetrahedral geometry.

\subsection{Spectroscopy}

In the IR spectra, the $\mathrm{C}-\mathrm{H}$ stretching frequencies of the pyrazolyl groups in complexes 1,2 and 3 are at 3101,3107 and $3105 \mathrm{~cm}^{-1}$, respectively. Complex 1 has a sharp peak at $3489 \mathrm{~cm}^{-1}$, indicative of the $\mathrm{O}-\mathrm{H}$
Table 6

Final atomic positional parameters of non-hydrogen atoms for complex 2

\begin{tabular}{|c|c|c|c|c|}
\hline Atom & $x$ & $y$ & $z$ & $\begin{array}{l}B_{\mathrm{eq}}{ }^{8} \\
\left(\AA^{2}\right)\end{array}$ \\
\hline $\mathrm{Cu}$ & $0.49236(11)$ & $0.08448(9)$ & $0.17157(9)$ & $2.14(3)$ \\
\hline $\mathrm{Cl}(1)$ & $0.65506(23)$ & $0.10904(19)$ & $0.01433(19)$ & $2.84(7)$ \\
\hline $\mathrm{Cl}(2)$ & $0.6534(3)$ & $-0.07496(23)$ & $0.28529(22)$ & $3.76(9)$ \\
\hline$N(1)$ & $0.5814(7)$ & $0.2899(6)$ & $0.2946(6)$ & $2.49(25)$ \\
\hline$N(2)$ & $0.5446(8)$ & $0.4276(6)$ & $0.2681(6)$ & $2.7(3)$ \\
\hline$N(3)$ & $0.1421(7)$ & $0.1564(6)$ & $0.2022(6)$ & $2.35(25)$ \\
\hline$N(4)$ & $0.2943(7)$ & $0.1163(6)$ & $0.2590(6)$ & $2.23(24)$ \\
\hline$C(1)$ & $0.6839(10)$ & $0.3263(8)$ & $0.4136(7)$ & $2.9(3)$ \\
\hline $\mathrm{C}(2)$ & $0.7192(10)$ & $0.4829(9)$ & $0.4667(8)$ & $3.6(4)$ \\
\hline$C(3)$ & $0.6279(10)$ & $0.5423(8)$ & $0.3703(9)$ & $3.5(4)$ \\
\hline $\mathrm{C}(4)$ & $0.4314(10)$ & $0.4361(8)$ & $0.1436(8)$ & $3.0(3)$ \\
\hline $\mathrm{C}(5)$ & $0.2605(9)$ & $0.4906(8)$ & $0.1658(7)$ & $2.6(3)$ \\
\hline $\mathrm{C}(6)$ & $0.2463(11)$ & $0.6512(8)$ & $0.2117(9)$ & $3.6(4)$ \\
\hline$C(7)$ & $0.0939(12)$ & $0.7098(8)$ & $0.2315(9)$ & $4.2(4)$ \\
\hline$C(8)$ & $-0.0417(11)$ & $0.6173(9)$ & $0.2057(9)$ & $3.9(4)$ \\
\hline $\mathrm{C}(9)$ & $-0.0316(10)$ & $0.4585(8)$ & $0.1581(8)$ & $3.2(4)$ \\
\hline$C(10)$ & $0.1221(9)$ & $0.3965(7)$ & $0.1387(7)$ & $2.4(3)$ \\
\hline$C(11)$ & $0.1192(9)$ & $0.2210(7)$ & $0.0876(7)$ & $2.5(3)$ \\
\hline $\mathrm{C}(12)$ & $0.0365(9)$ & $0.1556(8)$ & $0.2849(9)$ & $3.2(4)$ \\
\hline$C(13)$ & $0.1183(10)$ & $0.1167(8)$ & $0.3959(8)$ & $3.1(3)$ \\
\hline$C(14)$ & $0.2780(9)$ & $0.0933(8)$ & $0.3741(7)$ & $2.6(3)$ \\
\hline
\end{tabular}

${ }^{\mathrm{a}} B_{\mathrm{c}}$ is the mean of the principal axes of the thermal ellipsoid.

Table 7

Final atomic positional parameters of non-hydrogen atoms for complex 3

\begin{tabular}{lllll}
\hline Atom & $x$ & $y$ & $z$ & $\begin{array}{l}B_{\text {eq }}{ }^{a} \\
\left(\AA^{2}\right)\end{array}$ \\
\hline $\mathrm{Co}$ & $0.72079(4)$ & $0.14583(3)$ & $0.22677(3)$ & $3.163(18)$ \\
$\mathrm{Cl}(1)$ & $0.78447(8)$ & $0.12716(7)$ & $0.39909(5)$ & $4.59(4)$ \\
$\mathrm{Cl}(2)$ & $0.67100(7)$ & $0.32533(6)$ & $0.16620(6)$ & $4.62(4)$ \\
$\mathrm{N}(1)$ & $0.55060(21)$ & $0.05449(18)$ & $0.15951(16)$ & $3.11(11)$ \\
$\mathrm{N}(2)$ & $0.52395(21)$ & $-0.05766(19)$ & $0.17171(17)$ & $3.25(11)$ \\
$\mathrm{N}(3)$ & $0.95831(21)$ & $0.01072(18)$ & $0.20255(16)$ & $3.16(11)$ \\
$\mathrm{N}(4)$ & $0.86936(20)$ & $0.09955(17)$ & $0.17089(15)$ & $2.78(10)$ \\
$\mathrm{C}(1)$ & $0.4370(3)$ & $0.09522(24)$ & $0.08809(21)$ & $3.52(14)$ \\
$\mathrm{C}(2)$ & $0.3391(3)$ & $0.0102(3)$ & $0.05486(21)$ & $3.82(14)$ \\
$\mathrm{C}(3)$ & $0.3974(3)$ & $-0.08530(25)$ & $0.10919(23)$ & $3.88(14)$ \\
$\mathrm{C}(4)$ & $0.6268(3)$ & $-0.13285(24)$ & $0.24284(20)$ & $3.69(14)$ \\
$\mathrm{C}(5)$ & $0.7062(3)$ & $-0.20223(23)$ & $0.18868(21)$ & $3.43(14)$ \\
$\mathrm{C}(6)$ & $0.6395(3)$ & $-0.2964(3)$ & $0.1305(3)$ & $4.66(17)$ \\
$\mathrm{C}(7)$ & $0.7008(3)$ & $-0.3634(3)$ & $0.07590(25)$ & $5.25(18)$ \\
$\mathrm{C}(8)$ & $0.8323(3)$ & $0.3373(3)$ & $0.07915(23)$ & $4.94(18)$ \\
$\mathrm{C}(9)$ & $0.9007(3)$ & $-0.2461(3)$ & $0.13778(23)$ & $3.94(15)$ \\
$\mathrm{C}(10)$ & $0.8407(3)$ & $-0.17811(22)$ & $0.19388(20)$ & $3.23(13)$ \\
$\mathrm{C}(11)$ & $0.9299(3)$ & $-0.08494(23)$ & $0.26187(20)$ & $3.45(13)$ \\
$\mathrm{C}(12)$ & $1.0606(3)$ & $0.0207(3)$ & $0.16326(24)$ & $4.18(17)$ \\
$\mathrm{C}(13)$ & $1.0375(3)$ & $0.1170(3)$ & $0.10421(22)$ & $4.03(16)$ \\
$\mathrm{C}(14)$ & $0.9180(3)$ & $0.16354(23)$ & $0.11044(19)$ & $3.27(13)$ \\
\hline
\end{tabular}

${ }^{a} B_{\text {eq }}$ is the mean of the principal axes of the thermal ellipsoid.

stretching of the solvent $\left(\mathrm{CH}_{3} \mathrm{OH}\right)$. The $\mathrm{C}=\mathrm{N}$ stretching frequencies in complexes 1,2 and $\mathbf{3}$ are at 1506, 1512 and $1509 \mathrm{~cm}^{-1}$, respectively. All of the absorption spectra of complexes $\mathbf{1}$ and $\mathbf{2}$ in ace tonitrile are identical, 


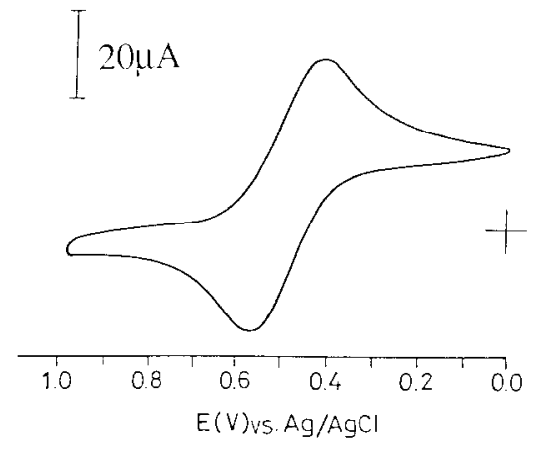

Fig. 4. Cyclic voltammetry for $2.0 \times 10^{-3} \mathrm{M}$ complex 1 in $\mathrm{CH}_{3} \mathrm{CN}$, containing $0.1 \mathrm{M}$ TBAP at room temperature. Scan rate $100 \mathrm{mV}$ $\mathbf{s}^{-1}$.

and the spectra exhibit a copper(II)-to-nitrogen chargetransfer band at $306 \mathrm{~nm}\left(\varepsilon=3710 \mathrm{M}^{-1} \mathrm{~cm}^{-1}\right)$ and another copper(II)-to-chloride charge-transfer band at $384 \mathrm{~nm}\left(\varepsilon=2420 \mathrm{M}^{-1} \mathrm{~cm}^{-1}\right)$.

The X-band powder ESR spectra were measured at room temperature. Complexes 1 and 2 both show an axial signal [17] $\left(g_{\|}=2.243, g_{\perp}=2.112\right.$ for 1 and $g_{\|}=2.280$, $g_{\perp}=2.046$ for 2). Complex 3 exhibits no ESR signal at room temperature. Nevertheless, there is a very broad isotropic signal at $77 \mathrm{~K}$, and the $g$ value is approximately 3.813 .

\subsection{Magnetic moments}

The magnetic moments were measured at room temperature. The magnetic moments of complex 1 $\left(\mu_{\mathrm{eff}}=1.85 \mathrm{BM}\right)$ can be regarded as normal. The value $\left(\mu_{\text {eff }}=1.82 \mathrm{BM}\right.$ per copper) for complex 2 indicates that the bridging $\mathrm{CuCl}_{2} \mathrm{Cu}$ unit has no strong magnetic interaction [17]. The magnetic moment of complex 3 $\left(\mu_{\mathrm{eff}}-4.60 \mathrm{BM}\right)$ shows that the tetrahedron cobalt(II) ion ( $\mathrm{d}^{7}$ configuration) is high-spin $(S=3 / 2)$ [18].

\subsection{Electrochemistry}

The redox properties of complexes 1 and 2 were studied using cyclic voltammetry (CV). All the CVs for complexes $\mathbf{1}$ and $\mathbf{2}$ in acetonitrile are identical. The $\mathrm{CV}$ for complex 1 in acctonitrilc shows a quasi-reversible wave, of which the $E_{1 / 2}$ is at $0.49 \mathrm{~V}$ versus $\mathrm{Ag} / \mathrm{AgCl}$ (see Fig. 4). Analysis of the peak current and scan rate by the Randle-Sevcik equation [19] indicates a one-electron transfer for this $\mathrm{Cu}(\mathrm{II}) / \mathrm{Cu}(\mathrm{I})$ redox couple.

\section{Acknowledgement}

This work was supported by the National Science Council, Taiwan, ROC.

\section{References}

[1] F.S. Keij, J.G. Haasnoot, A.J. Oosterling, J. Reedijk, C.J. O'Connor, J.H. Zhang and A.L. Spek, Inorg. Chim. Acta, 181 (1991) 185.

[2] S.M. Morehouse, A. Polychronopoulou and G.J.B. Williams, Inorg. Chem., 19 (1980) 3558.

[3] H.M.I. Hendriks, P.J.M.W.L. Birker, G.C. Verschoor and J. Reedijk, J. Chem. Soc., Dalton Trans., (1982) 623.

[4] F. Abraham, M. Lagrenee, S. Sueur, B. Mernari and C. Bremard, J. Chem. Soc., Dalton Trans., (1991) 1433.

[5] (a) N.J. Blackburn, R.W. Strange, A. Farooq, M.S. Haka and K.D. Karlin, J. Am. Chem. Soc., 110 (1988) 4263; (b) K.D. Karlin, P. Ghosh, R.W. Cruse, A. Farooq, Y. Gultneh, R.R. Jacobson, N.J. Blackburn, R.W. Strange and J. Zubieta, J. Am. Chem. Soc, 110 (1988) 6769; (c) N. Kitajima, T. Koda, S. Hashimoto, T. Kitagawa and Y. Moro-oka, J. Am. Chem. Soc., 113 (1991) 5664.

[6] (a) T.N. Sorrell, M.L. Garrity and D.J. Ellis, Inorg. Chim. Acta, 166 (1989) 71; (b) T.N. Sorrell and V.A. Vankai, Inorg. Chem., 29 (1990) 1687; (c) T.N. Sorrell, V.A. Vankai and M.L. Garrity, Inorg. Chem., 30 (1991) 207.

[7] F.S. Keij, R.A.G. de Graaff, J.G. Haasnoot, J. Reedijk and F. Pedersen, Inong. Chim. Acta, 156 (1989) 65.

[8] R.R. Hree, T. Enkoji and J.P. Dailey, J. Am. Chem. Soc., 79 (1957) 4230.

[9] E.J. Gabc, Y. Lc Page, P.S. Whitc and F.L. Lcc, Acta Crystallogr., Sect. A, 43 (1987) S294.

[10] Intemational Tables for X-ray Crystallography, Vol. 4, Kynoch, Birmingham, UK, 1974.

[11] W.G. Haanstra, W.A.J.W. van der Donk, W.L. Driessen, J. Reedijk, J.S. Wood and M.G.B. Drew, J. Chem. Soc., Dalton Trans., (1990) 3123.

[12] W.G. Haanstra, W.L. Driessen, R.A.G. de Graaff, J. Reedijk, Y.F. Wang and C.H. Stam, Inorg. Chim. Acta, 186 (1991) 215.

[13] A.W. Addison, T.N. Rao, J. Reedijk, J. van Rijn and G.C. Verschoor, J. Chem. Soc., Dalton Trans., (1984) 1349.

[14] (a) W.E. Marsh, W.E. Hatfield and D.J. Hodgson, Inorg. Chem., 21 (1982) 2679; (b) W.E. Marsh, D.S. Eggleston, W.E. Hatfield and D.J. Hodgson, Inorg. Chim. Acta, 70 (1983) 137; (c) W.E. Marsh, K.C. Patel, W.E. Hatfield and D.J. Hodgson, Inorg. Chem., 22 (1983) 511.

[15] W.G. Haanstra, W.L. Driessen, J. Reedijk, R. Frohlich and B. Krebs, Inorg. Chim. Acta, 185 (1991) 175.

[16] J. van Rijn, E. Bouwman, J.R. Empfield, W.L. Driessen and J. Reedijk, Polyhedron, 8 (1989) 1965.

[17] B.J. Hathaway and D.E. Billing, Coord. Chem. Rev., 5 (1970) 143.

[18] B.N. Figgis and R.S. Nyholm, J. Chem. Soc., (1959) 338; B.J. Hathaway and D.G. Holah, J. Chem. Soc., (1964) 2400.

[19] A.J. Bard and L.R. Faulkner, Electrochemical Methods: Fundamentals and Applications, Wiley, New York, 1980, p. 218 